Indonesian Journal of Physics and Nuclear Applications

Volume 2, Number 1, February 2017, p. 20-33

ISSN 2549-046X, (c) FSM UKSW Publication

\title{
Assessment of Analytical Instrumentation for Boron Measurement in BNCT System
}

\author{
Isman Mulyadi Triatmoko a and Sutjipto ${ }^{a}$ \\ ${ }^{\mathrm{a} C e n t r e}$ of Science and Accelerator Technology-BATAN J1. Babarsari, Yogyakarta, \\ 55281 Indonesia
}

\begin{abstract}
The methods boron measurement in BNCT system has generally progressed with developments in analytical instrumentation. Spectrophotometric methods remained the methods of choice for most routine applications until the development of ICP-OES. ICP-OES was also not adequately sensitive for nutritional and medical research involving animal tissues that are naturally low in boron (B). The development of plasma-source MS (e.g., ICP-MS) not only has overcome most of these drawbacks, but also its capability of measuring B isotopes. The application of nuclear reaction methods (mainly prompt- $\gamma$ spectrometry) has remained limited to some specialized fields. The validity and comparability of three different analytical techniques (QNCR, PGAA, and ICP-MS) for boron measurement in biological samples and application of these methods for examination of blood and tissue samples from a clinical study on boron uptake in blood, tissue, and neoplastic tissue, after infusion of BPA. The PET-based approach to TPS has been applied in BDTPS and a preliminary evaluation of the correct operation has been performed using a heterogeneous boron phantom, called HEBOM. The validation has been accompanied by calculations done with SERA, following the standard approach. BDTPS needs further in vivo experimental validations.
\end{abstract}

Keywords : BNCT, QNCR, PGAA, ICP-MS, PET, BDTPS

\section{INTRODUCTION}

Boron plays also a role in life sciences, though its biological and physiological potential has not been fully explored yet. For example, in animal models, boron was found to ameliorate fulminant hepatic failure by counteracting the changes associated with the oxidative stress. In cancer therapy, boron is mostly associated with BNCT, though more recently, the use of polyhedral boron compounds was proposed as carriers for radionuclide therapy and diagnostics Sommer AL, Lipman CB Bregadze VI,et al. Polyhedral heteroboranes have been the subject of intense research for over 40 years. A subset of this extensive class of compounds are dicarba-closo-dodecaboranes, commonly referred to as carboranes (an abbreviation of the IPUAC name carbaboranes) having the general formula C2B10H12. Radiopharmaceuticals were assessed by M.F. Hawthorne, A. Maderna, polymers were assessed by D.K. McLemore, et al, and a assortment of unique coordination compounds were assessed by $\mathrm{M}$. Westerhausen, et al. The initial attraction to carboranes for medicinal chemistry research was a result of their high boron content and stability to catabolism, which are important criteria for BNCT agentts. Boron is also used as a source of short range $\alpha$ (alpha) particles in cancer treatment using Boron Neutron Capture Therapy (BNCT) were assessed by Raaijmakers, C. P, et al.

BNCT is a novel technique for the treatment of cancer that uses ${ }^{10} \mathrm{~B}$-labeled compounds and neutron radiation to kill cancerous cells. The significance of $\mathrm{B}$ compounds in BNCT sistems from high neutron 
cross section or capture probability (3838 barns) of the ${ }^{10} \mathrm{~B}$ atom compared to other biologically ubiquitous atoms such as carbon (0.003 barns), hydrogen ( 0.33 barns), nitrogen (1.8 barns) and oxygen (0.0002 barns) atoms. The short range $(10 \mu \mathrm{m})$ cytotoxic $\alpha$ (alpha) radiation released in the neutron capture reaction kills the targeted cancerous cells without affecting the neighboring healthy cells were assessed by Moore, D. E. The most important requirements for a BNCT delivery agent are: 1.) low toxicity and normal tissue uptake, with a tumor:normal tissue and tumor:blood $(\mathrm{T}: \mathrm{Bl})$ boron concentration ratios of $\sim 3$; 2.) tumor boron concentration of $\sim 20 \mu \mathrm{g} \quad{ }^{10} \mathrm{~B} / \mathrm{g}$ tumor; 3.) relatively rapid clearance from blood and normal tissues, and persistence in tumor during neutron irradiations. The only two BNCT delivery agents currently used in clinical trials are sodium mercaptoundecahydro-closododecaborate $\quad\left(\mathrm{Na}_{2} \mathrm{~B}_{12} \mathrm{H}_{11} \mathrm{SH}\right)$, commonly known as sodium borocaptate (BSH), and the boron-containing amino acid (L)-4dihydroxy-borylphenylalanine, known as boronophenylalanine or BPA. Neither of these agents adequately fulfills the criteria indicated above, for this reason third generation agents incorporating one or more polyhedral borane anions or carboranes was proposed as carriers for radionuclide therapy and diagnostics in cancer therapy associated with BNCT.

Precise measurement of $\mathrm{B}$ and its isotopes is necessary for the evaluation of the tumor specificity and pharmacokinetics of $\mathrm{B}$ compounds for BNCT. Often it is necessary to be able to measure B in very small samples (e.g., biopsy-needle samples) to make sure that the drugs are actually localized in target tissue before exposing the patient to neutron sources were assessed by Johnson, D. A, et al. Therefore, accurate measurement of the $\mathrm{B}$ concentration is very critical for these applications. The National Institute of Standard and Technology (NIST) certified Standard Reference Materials (SRMs) such as NIST-boric acid standard for B isotope ratio and NISTbotanical standards for total B concentrations are widely used for verifying the accuracy of a measurement. A small amount of siliceous or a calcareous mineral matter present in these SRMs may contribute to the errors that may not be resolved by analytical methods. Therefore, one must use caution to ensure that the heterogeneity found in SRMs is appropriately considered and dealt with were assessed by Lindstrom, R.M, et al. The purpose of this study is to assessment of analytical instrumentation for boron measurement in BNCT system.

\section{DISCUSSION}

\section{Spectrophotometric Methods}

\section{Plasma-Source Methods}

Introduction of plasmas as ionization sources and the development of plasma-source analytical instruments (plasma-source-OES and MS) provided higher sensitivity and lower detection capability for B measurement than was possible by spectrophotometric, flame AES/AAS, and time consuming nuclear methods. There are several types of plasma, namely, the direct current (DCP) were assessed by Urasa, I. T, the inductively coupled plasma (ICP) were assessed by Kempster, P. L, et al, the microwave induced plasma (MIP) were assessed by Evans, E. H, et al and the glow discharge plasma (GDP) were assessed by Sheppard, B. S, et al. Plasmas have been generated from a number of gases or their mixtures were assessed 
by Jarvis, K. E. et al; however, most commercial plasma-source instruments use an argon ICP (i.e., ICP generated from argon) for ionization. The plasma source instruments are of two kinds, based on the detection method they employ (a) plasma source optical emission spectrometry (OES) such as ICPOES and DCP-OES and (b) plasma-source mass spectrometry (MS) such as ICP-MS, DCP-MS, and microwave induced plasma (MIP)-MS. OES has also been called atomic emission spectrometry (AES).

Atomic spectrometric methods such as AES and AAS revolutionized the measurement of a large number of elements, but these methods were not very sensitive for the element B. As a result, spectrophotometric methods remained the methods of choice for most routine applications until the development of ICPOES. ICP-OES was also not adequately sensitive for nutritional and medical research involving animal tissues that are naturally low in B. Development of plasma-source MS (e.g., ICP-MS) not only has overcome most of these drawbacks, but also its capability of measuring $\mathrm{B}$ isotopes has made possible (1) B concentration measurement by isotope dilution, (2) verification of $\mathrm{B}$ concentration by isotope fingerprinting in routine analysis, and (3) measurement of total B concentration and $\mathrm{B}$ isotope ratio in the same run for biological tracer studies. Therefore, plasma source MS appears to be the method of choice among present-day technologies.

\section{ICP-MS}

The ICP-MS is often the method of choice over ICP-OES and spectrophotometric methods for B measurement. The advantages of ICP-MS over other methods are higher sensitivity, lower detection limits, and simultaneous measurement of ${ }^{10} \mathrm{~B}$ to ${ }^{11} \mathrm{~B}$ isotopic ratio and total $\mathrm{B}$ concentration in a sample. The ability of ICP-MS to measure B-isotope ratios renders this instrument especially suitable for biological B tracer studies. The reported detection limits are at the ppb level, e.g., one $\mathrm{ppb}$ to $3 \mathrm{ppb}$ in biological materials, $0.15 \mathrm{ppb}$ in saline waters were assessed by Gregoire, D. C, and $0.5 \mathrm{ppb}$ in human serum were assessed by Vanhoe, H,et al. The uniqueness of ICP-MS is also due to its capability to carry out B measurement by the isotope dilution method which is considered the most precise for quantitative measurement.

The external calibration with an internal standard is most widely used method for plasmasource OES and MS because of its simplicity and labor efficiency. Other methods, such as standard addition, addition calibration, and isotope dilution methods are less common and are generally employed to deal with difficult sample matrices or to improve precision. For the external calibration method, an internal standard as close as possible to the mass number of the determinand elements should be selected were assessed by Vanhaecke, F,et al. Beryllium is the closest in mass number to $\mathrm{B}$ and therefore it is the most commonly used internal standard for B measurement. Use of $\mathrm{Be}$ as an internal standard was effective in mitigating matrix interferences were assessed by Evans, S.; Krahenbuhl, U and corrected the matrix induced suppression of ${ }^{10} \mathrm{~B}$ signals $(85.6 \pm 5.2 \%$ recovery relative to $0.14 \%$ $\mathrm{HNO}_{3}$ ), yielding $99.6 \pm 2.5 \%$ relative signal intensities were assessed by Vanhoe, H.; Dams, $\mathrm{R}$.

The development a model to estimate the ${ }^{10} \mathrm{~B}$ concentration value from the counts associated to each voxel by the PET 
tomographer. The ${ }^{18} \mathrm{~F}-\mathrm{BPA}$ is infused into the patient, who later undergoes scanning. The PET scanner can detect the radiation emitted when a positron-electron annihilation reaction occurs and ascertains the location of this reaction.

The synthesis of the ${ }^{18} \mathrm{~F}-\mathrm{BPA}$ has induced several scientists to put their attention on a Positron Emission Tomography (PET) based on BNCT. In particular, at the Kyoto Prefectural University of Medicine were assessed by Imahori Y, et al (Japan) and contemporarily at the Departments of Radiobiology and Neurosurgery were assessed by Kabalka G W,et al, University of Tennessee Medical Centre (USA) two groups of scientists have independently labelled boronophenylalanine (BPA) with ${ }^{18} \mathrm{~F}$ atom (a positron emitter).

For an example picture related ICP-OES or ICP-MS and FBPA-PET see Figure 1.

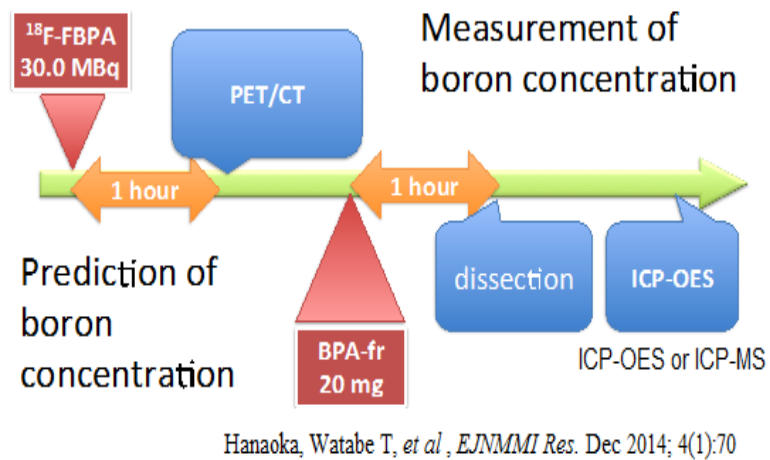

Figure 1. Measurement and Prediction of Boron Concentration

Spectral overlap of the ${ }^{12} \mathrm{C}$ peak on the ${ }^{11} \mathrm{~B}$ peak may interfere in ${ }^{11} \mathrm{~B} /{ }^{10} \mathrm{~B}$ ratio measurement if the sample solutions or the digest contains high levels of organic carbon as commonly found in microwave digests of biological materials. Evans and Krahenbuhl noted a significant interference of ${ }^{12} \mathrm{C}$ on $\mathrm{B}$ in the microwave digests of biological materials when the measurement was made in the normal resolution mode $(0.8 \mu)$. In the high resolution mode $(0.6 \mu)$ the effects depended on the sample types: the ${ }^{11} \mathrm{~B}$ peak of the hay sample was resolved completely, but those for the kidney and the flour sample were resolved only 75 and $55 \%$, respectively. An analyst should always watch for the possibility of error in B isotope ratio measurement due to anticipated enhancement in ${ }^{11} \mathrm{~B}$ signals by ${ }^{12} \mathrm{C}$. Where the isotope ratio measurement is not required, total $\mathrm{B}$ can be computed on the basis of ${ }^{10} \mathrm{~B}$ to avoid the error due to the overlap of ${ }^{12} \mathrm{C}$-peak. Spark source mass spectrometry (SSMS) is mainly used to determine concentrations of trace elements. Lukaszew et al. noted a satisfactory performance of SSMS for the measurement of isotopic composition. Secondary ion mass spectrometry (SIMS) employs sputtering of the surface atoms from small areas (typically, $250 \mathrm{x}$ $25 \mu \mathrm{m}$ ) of the sample by an energetic primary ion beam were assessed by Jones, L. E.; Thrower, P. A. The secondary ions generated from the sample are detected in a mass analyzer.

The applications of SIMS to BNCT were assessed by Moore. While SIMS can be used for quantitative B measurements in small samples also, it is particularly suitable for the measurement of intracellular B concentrations in BNCT. A very powerful tool for subcellular boron imaging is secondary ion mass spectrometry (SIMS). SIMS pictures image the boron distribution with a resolution of several nanometres, thus enabling the analyst to assess, whether the boron is found inside the cell nucleus, in the cytoplasm or in extracellular spaces were assessed by Smith DR, et al. For an example picture related SIMS see Figure $2 \mathrm{~A}$ and $\mathrm{B}$. 


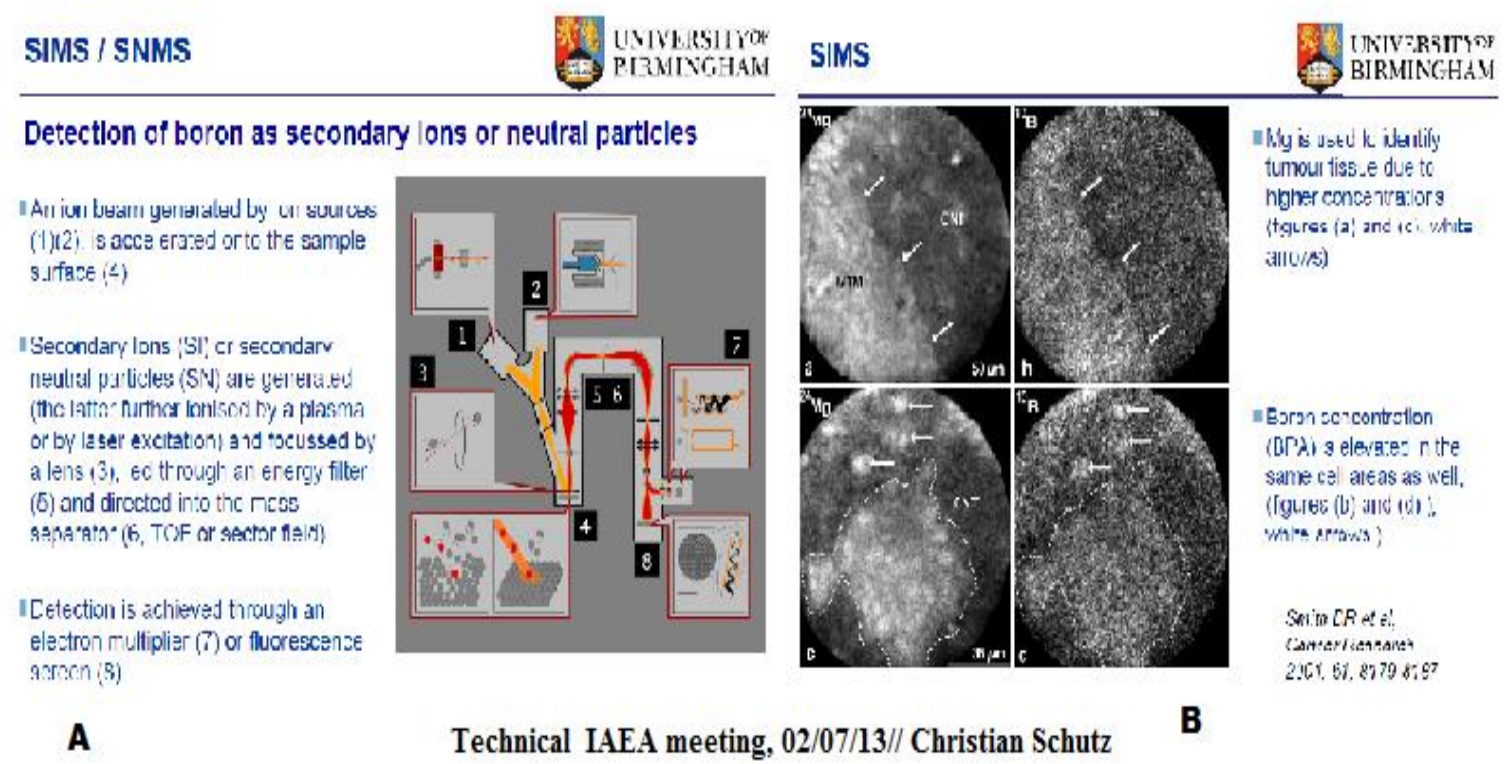

Figure 2. A. Devices of Secondary ion Spectrometry (SIMS) and B. Image SIMS

\section{Nuclear Reaction Analytical Methods}

\section{Neutron Activation Analysis}

In general, the sample is bombarded with neutrons, the elements of interest are made radioactive and quantity of the element is determined by measuring the radioactivity or radioactive decay products. NAA is a nondestructive method capable of handling solid samples with multi element detection capability and generally low detection limits. However, it is not suitable for sample mass or liquid volumes that pose a threat of radioactive leaks after activation. Ward et al. found good agreement between the value of 18 elements determined by NAA and ICPMS. The nuclear methods for $\mathrm{B}$ measurement were recently assessed by Pillay and Peisach. The measurements by the NAA methods require access to a nuclear reactor for the production of thermal neutrons for bombardment to convert isotope(s) of interest in a sample to radioisotopes were assessed by Johnson, P. E. Boron is an exception to this strategy. The activation of 10B by an incident beam of thermal neutrons does not make it radioactive but causes the following neutroncapture reaction:

${ }^{10} \mathrm{~B}+$ neutron $\rightarrow{ }^{\prime} \mathrm{Li}+\alpha$ particles $(2.31 \mathrm{MeV})+$ gamma-ray $(478 \mathrm{KeV})$

This reaction involves only the ${ }^{10} \mathrm{~B}$ isotope, which has approximately 20\% abundance in naturally occurring B. All NAA methods for B measurement are based on the measurement of one or more products ( $\alpha$-particles and $\gamma$-photons) of this reaction. Perhaps the most important method based on the measurement of $\alpha$ (alpha) particles is neutron activation (NA) MS while that based on the measurement of gama rays is prompt $\gamma$-ray spectrometry.

\section{Methods based on a particles}

Two methods detecting the fragments of the ${ }^{10} \mathrm{~B}(\mathrm{n}, \alpha)^{7} \mathrm{Li}$ reaction are neutron activation mass spectrometry (NA-MS) and quantitative neutron capture radiography (QNCR) were assessed by Gabel D, et al. In NA-MS the Henuclei emitted after neutron capture are detected 
directly by a mass spectrometer. quantitative application of neutron capture Measurements may be carried out with very low quantification limits, however, there may be a considerable contribution to the background signal by other isotopes emitting $\alpha$ - particles after neutron capture. QNCR is a radiography (NCR), which is used for the qualitative analysis of materials which emit charged particles upon neutron capture. For an example picture related NCR see Figure 3. A. B.
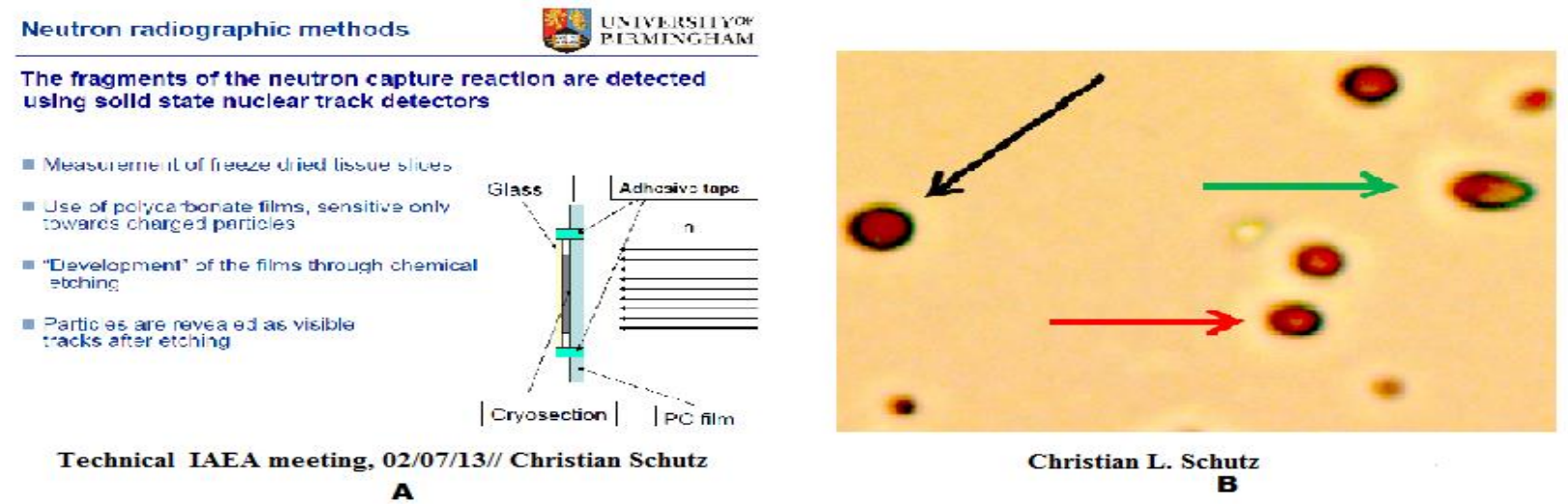

Figure 3. A. Devices of Neutron radiographic metthods and B. Alpha-and proton tracks of a radiographic image processing. The arrows all point to alpha tracks: there are examples given for track growth for $\delta=900$ (black) and $\delta \neq 900$ (red and green; with $\delta$ (green) $>\delta$ 9red). The image is scaled $48 \times 36 \mathrm{Qm}^{2}$

The protocol for neutron capture radiography in BNCT usually includes fixation of a piece of tissue on a SSNTD film, as the major objective of radiographic analysis is to image the boron distribution, therefore, any space left (or any "obstacle") between detector and sample would lead to a distorted picture. All radiographic images for the work presented were produced using a polyallyldiglycol-carbonate film purchased from TASTRAK (Track Analysis Systems Ltd., Bristol, United Kingdom). TASTRAK films are sensitive only towards irradiation of charged particle energies and fast neutrons.

For application of neutron radiography to analyse the boron content in tissue samples, tracks are almost exclusively produced by protons, ${ }^{4} \mathrm{He}^{-}$and ${ }^{7} \mathrm{Li}$ nuclei. Though the image for analysis is created by chemical etching, the actual evaluation of the data especially for quantitative analysis has to take place using a microscope and special imaging software. Contrary to NA-MS, analysis with QNCR is carried out using solid state nuclear track detectors (SSNTDs) enabling the user to spatially analyse the boron concentration in a sample by visualisation of tracks created by the impact of ${ }^{4} \mathrm{He}$ and ${ }^{7} \mathrm{Li}$ onto the STNTD's surface. Closely related to QNCR is spatially selective $\alpha$-spectrometry, using a different detector system.

Neutron activation mass spectrometry (NAMS): Iyengar et al. and Clarke et al. described an NA-MS method for simultaneous measurement of lithium and B in biological materials. The sample was placed in an ultrapure polyethylene "liner' and freeze-dried. The liners containing the freezedried samples were placed in lead containers and evacuated to about $1005 \mathrm{~Pa}$. The lead containers were pinched-sealed following 
evacuation for neutron irradiation. A static mass spectrometer was used to measure ${ }^{4} \mathrm{He}$ (from ${ }^{10} \mathrm{~B}$ ) and ${ }^{3} \mathrm{He}\left(\right.$ from ${ }^{6} \mathrm{Li}$ ) generated by the NA reaction. The error rate at $\geq 1 \mathrm{ppm} \mathrm{B}$ concentration was $1-5 \%$ but at $6 \mathrm{ppb}$ B the error rate increased to $75 \%$. This sensitivity is not adequate for BNCT and some nutritional and environmental applications.

\section{Methods based on the measurements of $\gamma$ - rays}

The most obvious advantage of such techniques over other techniques is that they are non-destructive. Several of these techniques are based on the on the high probability of ${ }^{10} \mathrm{~B}$ for neutron capture reactions, as the isotope has a cross section for thermal neutron of 3837 barn. The ${ }^{10} \mathrm{~B}(\mathrm{n}, \alpha)^{7} \mathrm{Li}$ reaction provides the possibility to detect two different kinds of fission products. Prompt-gamma activation analysis (PGAA, also PGNAA, PGRA or PGRS) is a form of gamma spectroscopy, which detects gamma rays emitted by short-live isotopes created by neutron capture. Neutron

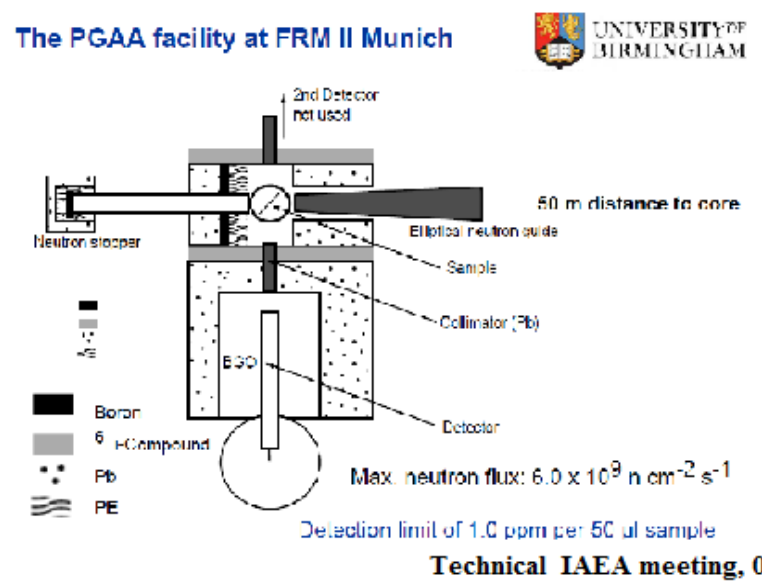

A

activation of the ${ }^{10} \mathrm{~B}$ istotope to ${ }^{11} \mathrm{~B} *$ is followed by fragmentation of the latter and by emission of a $478 \mathrm{keV}$ gamma photon in $94 \%$ of neutron capture reactions by the ${ }^{7} \mathrm{Li}$-nucleus. The spectroscopic recording of the gamma spectrum provides an indirect method to measure the integral boron content in a sample were assessed by Riley KJ, Harling OK.

Prompt $\gamma$ spectroscopy is an extensively used method for the measurement of ${ }^{10} \mathrm{~B}$ were assessed by Riley KJ, Harling OK. The $\gamma$-ray emitted from the disintegrating ${ }^{10} \mathrm{~B}$ nuclei due to the action of the neutron is detected. This is also a nondestructive method; however, it is not sensitive for the detection of low B levels (generally, < $5 \mu \mathrm{g} \mathrm{g}-1$ ) in the sample. As B concentration in the sample decreased, counting time to achieve desired precision increases logarithmically were assessed by Rogus, $\mathrm{R}$, et al. For example, counting time necessary to achieve $1 \%$ precision was $10 \mathrm{~h}$ for a sample containing one ppm B and $50 \mathrm{~h}$ for a sample containing 0.5 ppm B. For an example picture related PGAA see Figure 4 A and B.

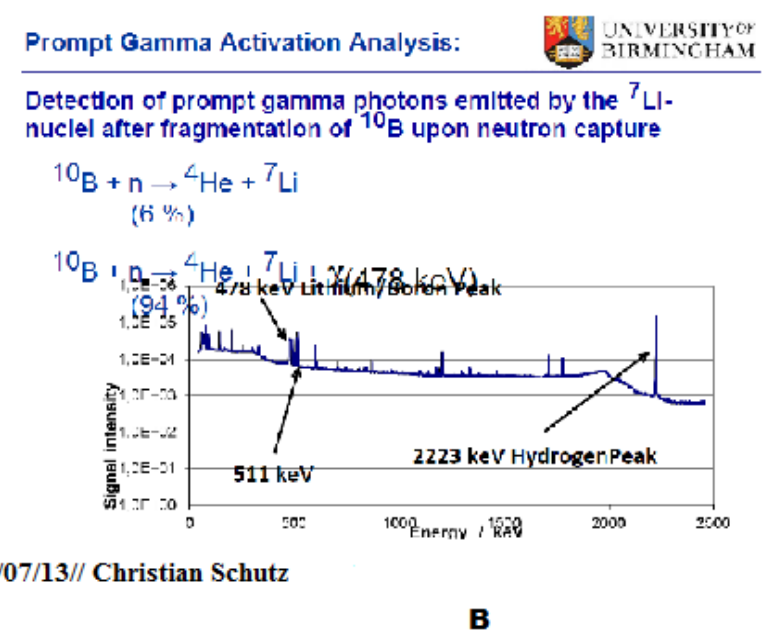

Figure 4. A. The PGAA facility at FRM II Munich and B. Promp Gamma Activation Analysis

For tumour samples, only QNCR was chosen, as it was the only method capable of performing locally selective boron measurement. For blood samples, first analysis by PGAA was performed, after which the same samples were used for subsequent analysis by 
ICP-MS. The only samples which were measured by all three methods were the biopsies from tumour free tissue.

First, cryosections for QNCR were prepared from the original piece of tissue taken after surgery. The remainder of the piece was then sent to Petten and Munich and measured by PGAA. After that, these samples were analysed by ICP-MS. For an example picture of the boron comparison of tissue samples using QNCR, PGAA and ICP MS see Figure 5.

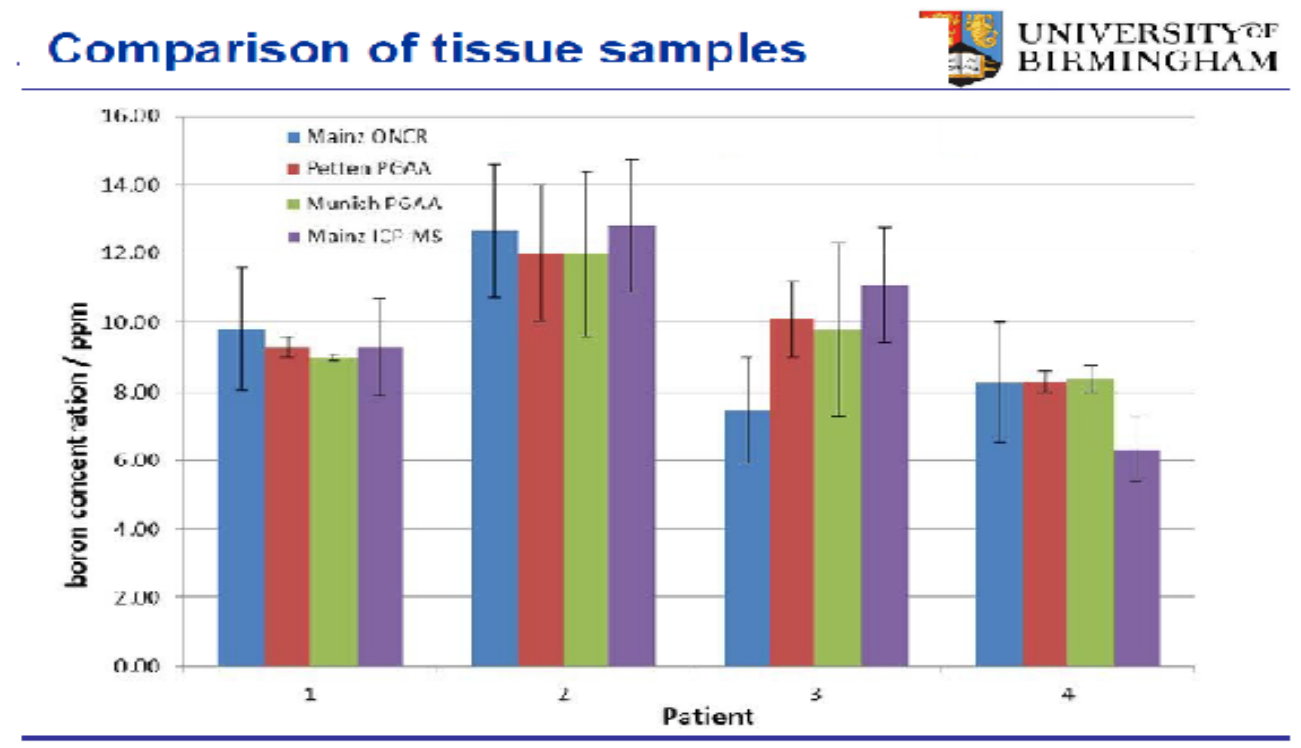

Technical IAEA meeting, 02/07/13// Christian Schutz

Figure 5. Comparison of tissue samples

The usual approach to validate analytical methods in direct comparison is to carry out measurements with all of the methods of a suitable standard reference material. One or several standard reference materials are measured by one or several analytical methods by a number of laboratories. The values have then to be collected and evaluated statistically to determine the conformity of each method against the others. Ideally, only the person collecting the data from each laboratory knows the content of the analyte to ensure the quality of such a trial. Such trials eventually yield individual information about the analytical quality of each participating laboratory, but they may also reveal, if there is a systematic error in boron measurement by one specific method (provided that such errors can be surely traced back to the method alone and not to sample preparation, the analyst, or the laboratory). To ensure the correctness (and, thus, their relevance for future research) of the data gathered from the samples taken during the clinical study, it was decided that the analytical methods used in this work had to be validated.

For the analytical methods chosen, there was no suitable standard reference material commercially available. This was particularly difficult, since QNCR allows only the measurement of solid samples, whereas ICP-MS can only process liquid or liquefied samples. Also, not only the mere boron measurement, but especially the boron measurement in a tissue matrix (all three methods) or blood matrix (ICPMS and PGAA) had to be carried out correctly. 
It was therefore decided to prepare an individual standard reference material for QNCR, which would later be measured by ICP-MS and QNCR.

After that, all samples from the clinical study would then be measured with the matching analytical method. Eventually for calibration of QNCR, standard reference samples of BPA in whole blood were prepared. The known boron concentration in these samples was measured by ICP-MS and PGAA to check both methods. In Figure 6 a schematic is shown illustrating the connections between measurements and samples, as well as the blood reference samples. Unfortunately, it was not possible to perform the sequence of analysis for all samples of tumour free tissue. Several biopsies were used up completely for preparation of the cryosections. Therefore, in order to increase statistics for the measurements with PGAA and ICP-MS, the smallest samples were used for PGAA analysis first. Since they had to be measured at room temperature, thawing made these samples completely ineligible for analysis by QNCR, so only analysis by ICP-MS was performed afterwards.

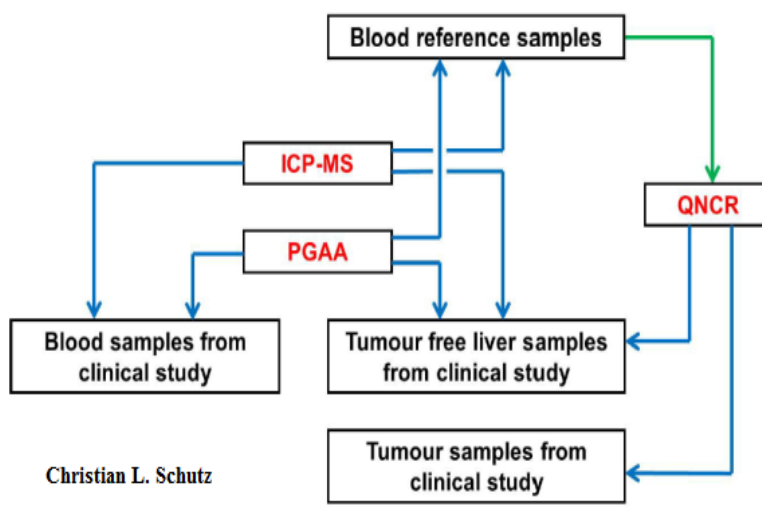

Figure 6. Connection between QNCR, PGAA and ICP-MS as methods for boron measurement, the samples obtained during the clinical study and blood reference samples used for calibration during analysis with QNCR. Blue arrows indicate measurement of samples by a specific method, the green arrow indicate that one set of samples was used for production of reference samples for QNCR.

\section{Boron Imaging}

Positron Emission Tomography (PET) is a functional imaging technique that allows the in vivo investigation of metabolic processes. This is made possible by linking a positron-emitting nucleus to the molecule of the substance (labelling) whose biological kinetics must be investigated.

These studies showed that the ${ }^{10} \mathrm{~B}$, used in BNCT, diffuses heterogeneously throughout the whole brain, without a clear distinction between tumour and healthy tissue. This consideration leads to a PET-based approach, that differs from the actual protocol based on the hypothesis of uniform two-zones boron distribution. In these studies important parameters for treatment planning have been achieved, such as the optimisation of the treatment time, the optimisation of the infusion protocol and the measurement of the cellular-level distribution of boron. Consequently, the idea to couple the treatment planning system with the boron distribution data acquired through PET scanning has been circulating in the BNCT scientific community. In particular, the BNCT Group at DIMNP of Pisa University (Italy) has collaborated with the Joint Research Centre (JRC) of Petten (The Netherlands) in order to design and implement this different approach to the TPS (Treatment Planning System). The idea was to link the PET data, related to the in vivo boron distribution, to the $3 \mathrm{D}$ modelling to make a realistic Monte Carlo (MC) simulation.

This idea led to the development of the original prototype software CARONTE were 
assessed by Cerullo $\mathrm{N}$, et al. This code contained a pre-processing module that received in input the PET images and prepared the input file for the MC simulation. Then a post-processing module provided some simple visualization tools for the analysis of the results. CARONTE was employed to carry out a comparative study between the standard and the PET based approach were assessed by Cerullo N, Daquino G G. However CARONTE built a simplified geometrical model of the patient's head for the MC simulation (a Snyder phantom, widely used in this field since early NCT TPS studies), in which the appropriate boron concentration value was computed at the location of each voxel. A natural follow up of the CARONTE experience was the improvement of the PET-based TPS methodology and the development of a real TPS, which contains both the standard tools and the connection to the boron data acquired through the PET scanning. The new TPS is called Boron Distribution Treatment Planning System (BDTPS) were assessed by Cerullo N, Daquino G G, Muzi L and is written in Visual $\mathrm{C}++$.

The standard approach in the TPS foresees the definition of the tumour and the healthy tissue regions on the CT or MRI images. A Monte Carlo model is automatically set up in order to perform the simulation of the treatment. At the moment, there are several TPS's in use in BNCT. Most of them make use of a Monte Carlo engine for the simulation of the nuclear reactions during the irradiation. For example, NCTPlan uses MCNP, SERA has rtt_MC in the background.

The default BDTPS calculations are referred to the main doses and fluences to take into account in BNCT:

- neutron fluence (thermal, epithermal, fast and total),

- photon fluence;

- boron dose;

- hydrogen neutron capture dose (or gamma dose);

- proton recoil dose;

- nitrogen dose

Figure 7. Shows the main components of the PET-based approach to TPS (BDTPS).

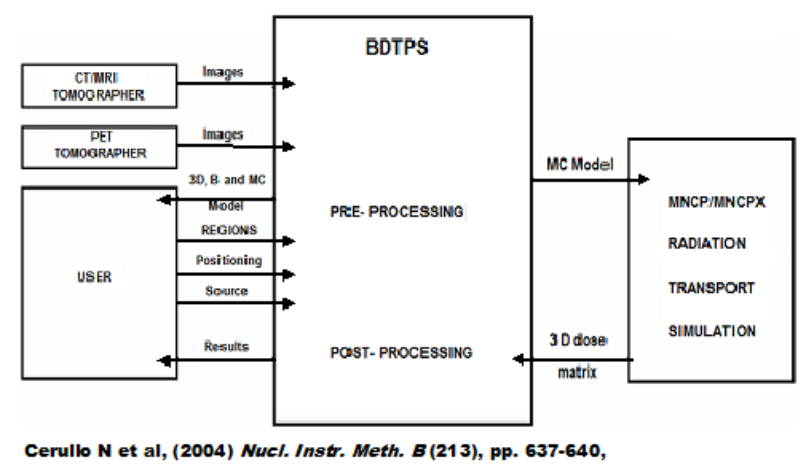

Figure 7. Main Components of PET-based Approach to TPS (BDTPS).

For an example picture related PET, MRI see Figure 8, $9 \mathrm{~A}$ and $\mathrm{B}$.

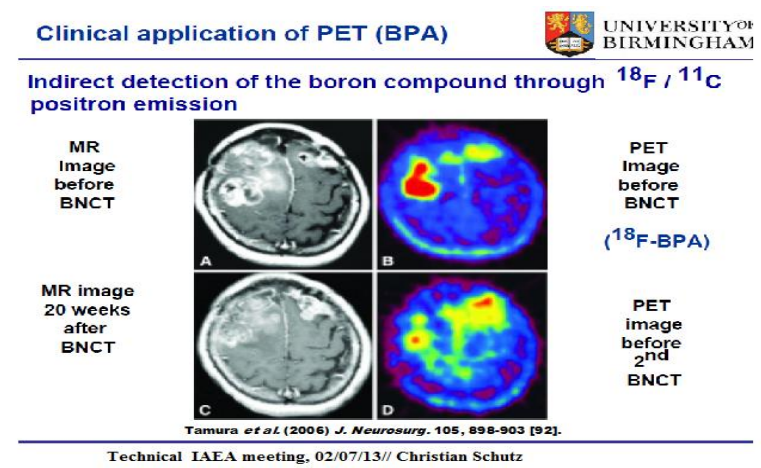

Figure 8. Clinical application of PET (BPA) 


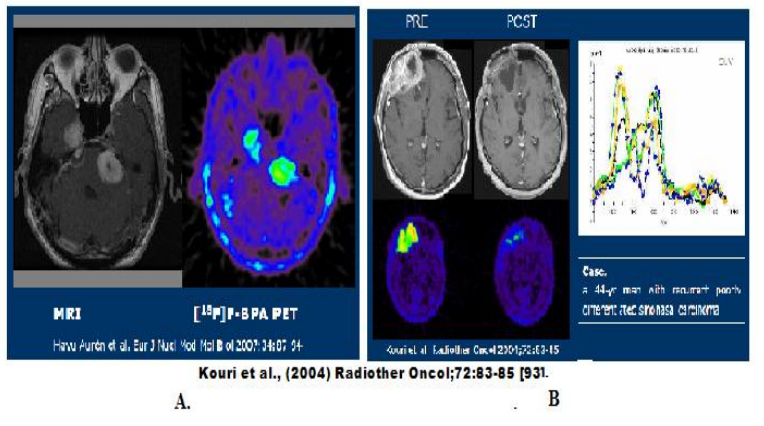

Figure 9. A. $\left[{ }^{18} \mathrm{~F}\right]$ F-BPA-PET in severe form of NF-2 and B. Novel target for BNCT

A preliminary validation of the methodology has been performed were assessed by Daquino $\mathrm{G} G$, et al using a special phantom HEBOM able to reproduce as much as possible the heterogeneous boron distribution. In this way, the validation exercise can be considered as close as possible to the real situation, which appears from the PET scanning. HEBOM's final design allows up to 64 vials to be placed in four layers, containing 8 different boron concentrations. Each vial has different colour depending on its boron content. The decision to colour the vials has been taken in order to facilitate quick vial recognition during the PET loading phase. The vials are located in the centre of the phantom. All the slabs contain clearance holes (15 $\mathrm{mm}$ diameter), between the vials, to host neutron and gamma detectors. Figure 10 shows HEBOM demounted (a) and mounted (b).

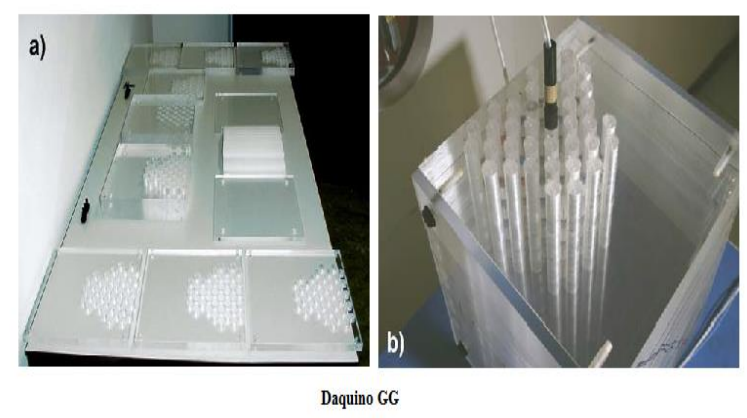

Figure 10. HEBON demounted (a) and (b) infront the HB11 aperture.
To provide the information necessary to reconstruct the $3 \mathrm{D}$ and the boron model in BDTPS, HEBOM had to be scanned with a CT and a PET tomographer.

\section{CONCLUSIONS}

The methods boron measurement in BNCT system has generally progressed with developments in analytical instrumentation. As a result, spectrophotometric methods remained the methods of choice for most routine applications until the development of ICP-OES. ICP-OES was also not adequately sensitive for nutritional and medical research involving animal tissues that are naturally low in B. Development of plasma-source MS (e.g., ICP-MS) not only has overcome most of these drawbacks, but also its capability of measuring $\mathrm{B}$ isotopes has made possible (1) B concentration measurement by isotope dilution, (2) verification of B concentration by isotope fingerprinting in routine analysis, and (3) measurement of total B concentration and $\mathrm{B}$ isotope ratio in the same run for biological tracer studies. Therefore, plasma source MS appears to be the method of choice among present-day technologies.

The application of nuclear reaction methods (mainly prompt- $\gamma$ spectrometry) has remained limited to some specialized fields. The validity and comparability of three different analytical techniques (QNCR, PGAA, and ICP$\mathrm{MS}$ ) for boron measurement in biological samples and application of these methods for examination of blood and tissue samples from a clinical study on boron uptake in blood, tissue, and neoplastic tissue, after infusion of BPA. Compare a locally selective method (QNCR) with two integral measuring methods (PGAA and ICP-MS) to yield results in good agreement for the same tissue samples containing boron of a confirmed morphology. 
The ongoing clinical study, a combination of computed QNCR with histological analysis was established as a suitable method to correlate the morphology of tissue and the boron concentration. The bulk sample analysis is not suitable to determine and to image the ${ }^{10} \mathrm{~B}$ concentration in tumour tissue, especially if characterisation of the uptake behaviour of BPA for future clinical application of BNCT is required.

The PET-based approach to TPS has been applied in BDTPS and a preliminary evaluation of the correct operation has been performed using a heterogeneous boron phantom, called HEBOM. The validation has been accompanied by calculations done with SERA, following the standard approach. BDTPS needs further in vivo experimental validations. The modelling will be performed with both BDTPS and SERA. This suggests that the possibility to use more realistic boron distribution maps acquired by means of PET imaging in BNCT treatment planning deserves more consideration and further investigation.

\section{REFERENCES}

Bregadze VI, Sivaev IB, Glazun SA (2006) Polyhedral Boron Compounds as Potential Diagnostic and Therapeutic Antitumor Agents. Anti-Cancer Agents in Medicinal Chemistry (Formerly Current Medicinal Chemistry - AntiCancer Agents) 6:75-109

Cerullo N, Daquino G G 2001 CARONTE (1998). A Treatment Planning System Nased on Real Macroscopic Boron Distribution and MCNP-4 ${ }^{\mathrm{a}}$ Code: Results and Discussion, Frontiers in Neutron Capture Therapy: Proc. 8th International Symposium on Neutron Capture Therapy (La Jolla, CA, USA,
13- 18 September 1998), Plenum Press, New York, Vol. 1, pp. 225-230

Cerullo N, Daquino G G, Moss R L, Muzi L 2002 Evaluation of the HeterogeneousBoron-Distribution Influence on the computed dose in BNCT Treatment Planning Systems, Research and Development in Neutron capture Therapy: Proc. 10th Int. Symp. on Neutron Capture Therapy for cancer, (Essen, Germany , 8-13 September 2002), eds W Sauerwein, R Moss A Wittig (Monduzzi Editore), pp. 541-545

Cerullo N, Daquino G G, Muzi L, Esposito J (2004) Nucl. Instr. Meth. B (213), pp. 637640, Jan. 2004

Chem. Int. Ed. Engl. 40 (2001) 1902.

Christian L. Schütz, (2012), Boron measurement in biological samples - Intercomparison of three analytical methods to assist development of a treatment protocol for neoplastic diseases of the liver with Boron Neutron Capture Therapy, Dissertation zur Erlangung des akademischen Grades, im Promotionsfach Chemie am Fachbereich Chemie, Pharmazie und Geowissenschaften der Johannes Gutenberg-Universität in Mainz

Christian L. Schütz, (2013), Boron Analysis and Boron Imaging in Boron Neutron Capture Therapy, IAEA Technical Meeting on Research Reactor Users' Networks (RRUNs): Advances in Neutron Therapy.

Clarke, W. B.; Koekebakker, M.; Barr, R. D.; Downing, R. G.; Fleming, R. F.(1987), Appl. Radiat. Isotopes (Int. J. Radiat. Appl. Instrum. Part A), 38, 735-747.

D.K. McLemore, D.A. Dixon, S.H. Strauss, Inorg. Chim. Acta 294 (1999) 193.

Daquino G G 2003 PET-based Approach to Treatment Planning Systems: an Improvement toward successful Boron Neutron Capture Therapy (BNCT) (Luxembourg: Office for Official Publications of the European Communities) 
Daquino G G, Cerullo C, Mazzini M, Moss R L, Muzi L 2004 Appl Radiat Isot. 61(5) 893-897.

Evans, E. H.; Caruso, J.(1993), A. J. Anal. Ato. Spectrom., 8, 427-431.

Evans, S.; Krahenbuhl, U.(1994) J. Anal. At. Spectrom., 9, 1249-1253.

Gabel D, Holstein H, Larsson B, Gille L, Ericson G, Sacker D, Som P, Fairchild RG (1987) Quantitative Neutron Capture Radiography for Studying the Biodistribution of Tumor-seeking Boron-containing Compounds. Cancer Res 47(20):5451-5454

Gregoire, D. C.(1990) J. Anal. Ato. Spectrom., 5, 623-626.

Hanaoka K, Watabe T, Naka S, et al, FBPA PET in boron neutron capture therapy for cancer: prediction of $\mathrm{B}$ concentration in tumor tissue an rat xenograft model. EJNMMI Res, Dec 2014;4(1): 70

Imahori Y, Ueda S, Ohmori Y, Kusuki T, Ono K, Fujii R, Ido T (1998) J. Nucl. Med. 39(2), 325-333

Jarvis, K. E.; Gray, A. L.; Houk,( 1992), R. S. Handbook of Inductively Coupled Plasma Mass Spectrometry.Chapman \& Hall, New York.

Johnson, D. A.; Siemer, D. D., Bauer, W. F.(1992) Anal. Chim. Acta, 270, 223230.

Johnson, P. E.(1989), J. Micronutrient Anal., $6,59-83$.

Jones, L. E.; Thrower, P. A. (1990), Carbon, 28, 239-241.

Kabalka G W, Smith G T, Dike J P, Reid W S, Longford C P D, Robert T G, Reddy N K , Buonocore E, Hübner K F (1997) The Journal of Nuclear Medicine 38 1762-1767

Kempster, P. L.; van Vliet, H. R.; van Staden, J. F.(1989), Anal. Chim. Acta, 218, 6976.

Lindstrom, R. M.; Byrne, A. R.; Becker, D. A.(1990); Smodis, B.; Garrity, K. M. Fresenius Z. J. Anal.Chem., 338, 569571.
Lukaszew, R. A.; Marrero, J. G.; Cretella, R. F.; Noutary, C. J.(1990), Analyst, 115, 915917.

M. Westerhausen, C. Gu“ ckel, S. Schneiderbauer, H. No" th, N.S. Hosmane, Angew.

M.F. Hawthorne, A. Maderna, Chem. Rev. 99 (1999) 3421.

Matsumoto, T.; Aizawa, O.(1990), Appl. Instrum. A, 41, 897-903.

Moore, D. E. (1990) J. Pharm. Biomed. Anal., $8,547-553$.

Moore, D. E. (1990) J. Pharm. Biomed. Anal., 8, 547-553.

Pillay, A. E.; Peisach, M.(1992), Nucl. Instrum. Method. Phys. Res. B., 66, 226-229.

Raaijmakers, C. P.; Konijnenberg, M. W.; Dewit, L.; Haritz, D.; Huiskamp, R.; Philipp, K.; Siefert, A.; Stecher-Rasmussen, F.; Mijnheer, B. J (1995). Acta Oncologica, $34,517-523$.

Riley KJ, Harling OK (1998) An improved prompt gamma neutron activation analysis facility using a focused diffracted neutron beam. Nuclear Instruments and Methods in Physics Research Section B: Beam Interactions with Materials and Atoms 143(3):414-421

Rogus, R.; Harling, O. K.; Olmez, I.; Wirdzek, S. (1992), Boron-10 prompt gamma analysis using diffracted neutron beam. In Progress in Neutron Capture Therapy for Cancer (B. J. Allen et al., Eds.), pp. 301304. Plenum New York.

Sheppard, B. S.; Caruso, J. A.(1994), J. Anal. Ato. Spectrom., 9, 145-149.

Smith DR, Chandra S, Coderre JA, Morrison GH (1996) Ion Microscopy Imaging of 10B from $\mathrm{p}$-Boronophenylalanine in a Brain Tumor Model for Boron Neutron Capture Therapy. Cancer Research 56(19):43024306

Urasa, I. T (1984). . Anal. Chem., 56, 904-908.

Vanhaecke, F.; Vanhoe, H.; Vandecasteele, C.; Dams, (1991), R. Anal. Chim. Acta, 244, 115-122. 
Vanhoe, H.; Dams, R.; Vandecasteele, C.; Versieck,(1993), J. Anal. Chim. Acta, 281, 401-411.

Vanhoe, H.; Dams, R.; Vandecasteele, C.; Versieck,(1993), J. Anal. Chim. Acta, 281, 401-411.

Ward, N. I.; Abu-Shakra, F. R.; Durrant, S.

F.(1990), Biol. Trace Element Res., 26, 177-187. 